\title{
Absolute determination of the effect of scattering and fluorescence on $x$-ray attenuation measurements
}

\author{
C Q Tran, M D de Jonge, Z Barnea and C T Chantler \\ School of Physics, University of Melbourne, Victoria 3010, Australia \\ E-mail: tran@physics.unimelb.edu.au
}

Received 21 April 2004

Published 20 July 2004

Online at stacks.iop.org/JPhysB/37/3163

doi:10.1088/0953-4075/37/15/011

\begin{abstract}
We investigate the effect of $\mathrm{x}$-ray scattering and fluorescence upon measurements of the x-ray mass attenuation coefficient. Measurements of scattering and fluorescence are obtained from a comparison of attenuation measurements using different sized apertures to admit varying amounts of the scattering and fluorescence into the detectors. The result of such a comparison is found to be in good agreement with a theoretical calculation of the fluorescent and scattered photons reaching the ion chambers and, under our experimental conditions, decreases the measured attenuation coefficients of silver by up to $0.2 \%$.
\end{abstract}

\section{Introduction}

Elastic and inelastic scattering processes in atoms, gases and elemental solids remain major topics of current research and investigation [1]. Major experimental coincidence studies [2, 3] provide valuable information about the relative magnitudes of individual processes and their relative angular dependence, but are difficult to perform and often appear to disagree with current theoretical predictions. Clearly, more work is needed in this experimental area [4-8]. New absolute measurements in this area will be valuable and are the subject of this paper.

Measurements of x-ray attenuation are affected by secondary photons reaching the detectors. These include incident photons scattered by the absorbing material and by the air path, as well as by x-ray fluorescence produced in the absorber by the incident beam. The magnitude of the contribution of these effects depends on the $\mathrm{x}$-ray optics, collimation, the photon energy, the detector response function and on the atomic number, quality and thickness of the absorbing sample. Although the secondary radiation (scattering and fluorescence) and its angular distribution can be observed using high-efficiency detectors at high-flux synchrotron sources, the effect is usually assumed to be small compared to the experimental errors and therefore neglected. At best a theoretically calculated correction is made. 
However, predictions of scattering cross-sections and of fluorescence vary by several per cent, depending on model assumptions, so the resulting value of the photoelectric mass absorption coefficient $[\mu / \rho]_{\text {pe }}$ is also uncertain by a corresponding amount. The effect from fluorescent photons can be significant, especially in the vicinity of absorption edges. Also, assumptions relating to coherent scattering processes (Rayleigh scattering for amorphous materials versus Laue-Bragg and thermal-difuse scattering for crystalline materials) have classified materials into two distinct groups: perfectly crystalline and perfectly amorphous. In practice, however, samples do not conform to these ideals [9, 10]. Since alternative assumptions lead to scattering components differing by some orders of magnitude, significant error is introduced, especially at high energies and for low $Z$ materials where the photoelectric cross-section $\sigma_{\mathrm{pe}}$ is comparable with the scattering cross-sections. Furthermore, at high energies, Rayleigh scattering becomes strongly peaked in the forward direction and must be carefully evaluated in order to obtain accurate results for both the total mass attenuation and the photoelectric absorption cross-sections.

Observation of the effect of scattering has been reported earlier [11]. The different magnitudes of the effects observed were used to identify the dominant coherent scattering modes, in the case of copper as Rayleigh scattering (R), where coherently scattered photons from atoms are assumed to be uncorrelated, and in the case of silicon as thermal diffuse scattering (TDS), where interference effects occur due to thermal vibration of atoms located at ideal crystalline positions. The former is roughly one order of magnitude larger than the latter.

In this paper we describe a development of the x-ray extended-range technique (XERT) $[12,13]$ in which we isolate and determine the effect of scattering and fluorescence at relatively high energies where contributions from these processes become significant. We apply the technique to attenuation measurements of silver in the broad energy range between $15 \mathrm{keV}$ and $50 \mathrm{keV}$. We show that the effects of scattering and fluorescence, observed at levels between $0.03 \%$ and $0.3 \%$ of the mass attenuation coefficient, are in agreement with theoretical predictions.

\section{Photon-atom interaction cross-sections}

The interaction between photons and atoms involves complex physical processes, especially in the vicinity of an absorption edge. Extensive discussions including historical notes on this topic can be found elsewhere $[14,15]$. In the intermediate energy region (from a few $\mathrm{keV}$ up to a few hundred $\mathrm{keV}$ ) and away from absorption edges, the total cross-section $\sigma_{\text {tot }}$ can be written as the sum of the cross-sections for the most probable individual processes

$$
\sigma_{\mathrm{tot}}=\sigma_{\mathrm{pe}}+\sigma_{\text {incoh }}+\sigma_{\mathrm{coh}}
$$

where $\sigma_{\mathrm{pe}}$ is the atomic photoelectric absorption cross-section, and $\sigma_{\mathrm{coh}}$ and $\sigma_{\text {incoh }}$ are the coherent and incoherent cross-sections respectively. Other processes contributing to the total cross-section, such as pair production, triplet production and photonuclear absorption, are significant only at energies $E>1 \mathrm{MeV}$ and are therefore omitted from this study.

In the atomic photoelectric absorption process a photon is totally absorbed by an atomic electron resulting in the emission of the electron. The created vacancy is subsequently filled, resulting in the possible emission of a fluorescence photon. As photoelectric absorption occurs only for atomic shells whose binding energies are lower than the incident photon energy, the function of $\sigma_{\mathrm{pe}}$ versus photon energy exhibits discontinuous jumps at absorption edge energies. 
The photoelectric absorption cross-section $\sigma_{\mathrm{pe}}$ is directly related to the imaginary component $f^{\prime \prime}$ of the atomic form factor by the optical theorem:

$$
f^{\prime \prime}=\frac{E \sigma_{\mathrm{pe}}}{2 h c r_{e}}
$$

where $E$ is the photon energy, $h$ and $c$ are Planck's constant and the speed of light and $r_{e}$ is the classical electron radius.

In general, coherent scattering may include, in addition to Rayleigh scattering, other components such as the pair production process. Similarly, incoherent scattering may include, in addition to Compton scattering, the triple production process. In the intermediate energy range, we can restrict the terms coherent or elastic to imply Rayleigh scattering except in the case of crystalline materials where Laue-Bragg and thermal-diffuse scattering occur [19-21]. Similarly, in the intermediate energy range, we can restrict the terms incoherent or inelastic to imply Compton scattering.

In Rayleigh scattering, photons are elastically scattered by bound electrons with the atom neither ionized nor excited. In this process, the incident photon is recoiled by the entire atom, changing its momentum and polarization while its energy remains unaltered. This scattering is 'coherent' (from different parts of the atomic charge distribution) and gives rise to interference effects. The scattering is forward peaked and can be calculated from [16]:

$$
\sigma_{R}=\pi r_{e}^{2} \int_{-1}^{1}\left(1+\cos ^{2} \theta\right) f^{2}(q, Z) \mathrm{d}(\cos \theta)
$$

where $f(q, Z)$ is the atomic form factor which depends on the atomic number $Z$ and on the momentum transfer $q=\sin (\theta / 2) / \lambda$, where $\theta$ is the scattering angle and $\lambda$ is the wavelength of the incident photons.

Compton scattering refers to a process where photons are inelastically scattered by atomic electrons without causing ionization, losing some of their energy and changing direction. This process dominates when the photon energy approaches the $100 \mathrm{keV}$ region. The Compton cross-section $\sigma_{C}$ can be calculated from

$$
\sigma_{C}=\pi r_{e}^{2} \int_{-1}^{1} \frac{1+\cos ^{2} \theta+\frac{k^{2}(1-\cos \theta)^{2}}{1+k(1-\cos \theta)}}{[1+k(1-\cos \theta)]^{2}} S(q, Z) \mathrm{d}(\cos \theta)
$$

where $k=\hbar \omega / m_{e} c^{2}$ and $S(q, Z)$ is the incoherent scattering function depending on the recoil momentum $q$ and the atomic number $Z[17,18]$.

For highly-ordered crystalline material interference effects occur between photons coherently scattered from planes of atoms resulting in Bragg diffraction. In this case, the contribution from the Rayleigh scattering term in equation (1) must therefore be replaced by the Laue-Bragg cross-section $\sigma_{\mathrm{LB}}$ or the thermal diffuse scattering cross-section $\sigma_{\mathrm{TDS}}$ :

$$
\sigma_{\mathrm{tot}}=\sigma_{\mathrm{pe}}+\sigma_{C}+\left\{\begin{array}{l}
\sigma_{\mathrm{LB}} \\
\sigma_{\mathrm{TDS}}
\end{array}\right.
$$

For a given photon energy, Laue-Bragg diffraction occurs only at particular orientations of the crystal relative to the incident photon and hence the term $\sigma_{\mathrm{LB}}$ should be omitted from equation (5) when the conditions for Laue-Bragg diffraction are not satisfied. In that case, the total coherent scattering is due to thermal vibrations which give rise to deviations from the ideal crystalline positions of the atoms. The thermal diffuse scattering cross-section $\sigma_{\mathrm{TDS}}$ 


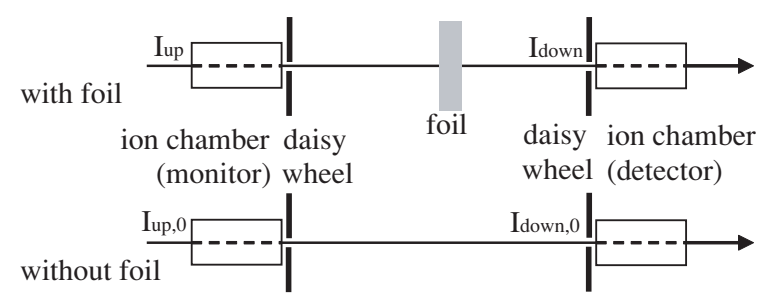

Figure 1. Experimental arrangement.

is much smaller than the Rayleigh scattering cross-section. Detailed formulations of $\sigma_{\mathrm{LB}}$ and $\sigma_{\text {TDS }}$ have been given elsewhere [19-21].

\section{Experimental details}

The experiment was conducted at the bending magnet beamline 1BM at the APS synchrotron at energies in the 15-50 keV range. Figure 1 shows key parts of the experimental arrangement used for the scattering and fluorescence investigations. Details of the experimental setup have been discussed elsewhere $[10,13]$. The beam was monochromatized by reflection from the planes of a double-crystal (400) silicon monochromator and was collimated to a $1 \times 1 \mathrm{~mm}^{2}$ cross-section before entering the upstream monitor ion chamber.

Two nitrogen gas flow ion chambers were employed to simultaneously measure the intensities of the incident (monitor) and the attenuated (detector) beams. Ten repeated measurements of one second exposure time were taken for each data point. The count rate was typically $2-5 \times 10^{5}$ counts per second for the unattenuated beam.

At each energy we used three foils whose thicknesses cover a large range of attenuation. The foils were mounted on a sample stage which was translated to move the foils in and out of the beam, and rotated to align the foil perpendicular to the beam. Between the ion chambers and the sample stage there were two 'daisy wheels' [31] made of $2 \mathrm{~mm}$ thick copper. Near the rim of each daisy wheel there were three circular apertures with diameters of $3 \mathrm{~mm}, 6 \mathrm{~mm}$ and $16 \mathrm{~mm}$. With the distance between the ion chambers and the specimen being $295 \mathrm{~mm}$, these apertures define cones with opening half-angles of 5, 10 and $27 \mathrm{mrad}$, corresponding to solid angles of $8.1 \times 10^{-5}$ sterad $3.2 \times 10^{-4}$ sterad and $2.3 \times 10^{-3}$ sterad, respectively. The attenuation measurements were carried out with all three apertures in turn at each energy to admit different amounts of fluorescent and scattered photons into the detectors.

In this experimental configuration, the measured mass attenuation coefficient $[\mu / \rho]_{\text {meas }}$ is $[10,13]$

$$
[\mu / \rho]_{\text {meas }}=-\frac{1}{\rho t} \ln \left(\frac{\frac{I_{\text {down }}}{I_{\text {up }}}}{\frac{I_{\text {down }, 0}}{I_{\text {up }, 0}}}\right)
$$

where $(\rho t)$ is the local mass per unit area of the specimen; $I_{x}$ represents the readings of the ion chambers as labelled in figure 1; the subscripts 'down' and 'up' indicate the downstream or the upstream ion chamber with the sample inserted in the beam, respectively; the subscripts 'down, 0' and 'up, 0' indicate the downstream and the upstream ion-chamber readings with the sample removed from the beam.

The ion chamber readings in equation (6) must be differentiated from the beam intensities for the modelling of the effect as discussed later in equation (7). Also, $[\mu / \rho]_{\text {meas }}$ in 


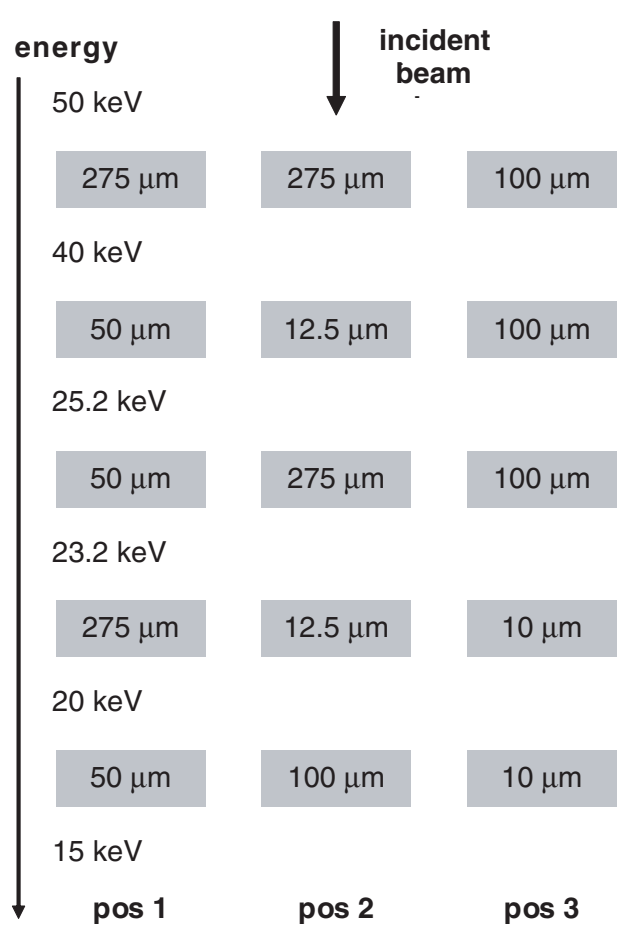

Figure 2. Thicknesses and positions of silver foils used at different energies.

equation (6) is the mass attenuation coefficient measured in the presence of secondary photons from the fluorescence, from scattering by the absorbing sample (equation (5)), as well as photons scattered in the passage of the beam through air and through the Kapton detector windows. Some fraction of these secondary photons reach the ion chambers through the solid angles defined by the daisy-wheel apertures. A key point of our experiment is the comparison of the mass attenuation coefficients measured in turn with the three aperture sizes. From this comparison we determine the absolute values of the fluorescence and scattering contributions to the attenuation measurement.

Our samples were high-purity $(99.999 \%)$ silver foils with nominal thicknesses ranging from $10 \mu \mathrm{m}$ to $275 \mu \mathrm{m}$ supplied by the Goodfellow and ESPI companies. Figure 2 shows the nominal thicknesses of the three foils used in each energy range. The three positions indicated in this figure correspond to the three positions of the sample stage, each of which can be moved into the beam during the experiment. The attenuation cross-sections were measured with the largest and the smallest apertures for foils at the first position, and with the largest and the medium apertures for foils at the second and third positions. These combinations of foils and aperture sizes provided us with an extensive set of data for scattering investigations.

\section{Modelling of the effects of scattering and fluorescence}

\subsection{Explicit representation of scattering and fluorescence}

To model the mass attenuation coefficients $[\mu / \rho]_{\text {meas }}$ of equation $(6)$, we introduce parameters representing contributions from fluorescence and scattering explicitly: 


$$
\begin{aligned}
{[\mu / \rho]_{\text {meas }} } & =-\frac{1}{\rho t} \ln \left(\frac{\frac{I_{\text {down }}}{I_{\text {up }}}}{\frac{I_{\text {down }, 0}}{I_{\text {up }, 0}}}\right) \\
& =-\frac{1}{(\rho t)} \ln \left(\frac{\frac{a\left(I+\Delta I_{F}+\Delta I_{R}+\Delta I_{C}\right)}{b\left(I_{0}+\Delta I_{0, F}+\Delta I_{0, R}+\Delta I_{0, C}\right)}}{\frac{a\left(I^{\prime}+\Delta I_{F}^{\prime}+\Delta I_{R}^{\prime}+\Delta I_{C}^{\prime}\right)}{b\left(I_{0}^{\prime}+\Delta I_{0, F}^{\prime}+\Delta I_{0, R}^{\prime}+\Delta I_{0, C}^{\prime}\right)}}\right) \\
& =-\frac{1}{(\rho t)} \ln \left(\frac{\frac{\left(I+\Delta I_{F}+\Delta I_{R}+\Delta I_{C}\right)}{\left(I_{0}+\Delta I_{0, F}+\Delta I_{0, R}+\Delta I_{0, C}\right)}}{\frac{\left(I^{\prime}+\Delta I_{F}^{\prime}+\Delta I_{R}^{\prime}+\Delta I_{C}^{\prime}\right)}{\left(I_{0}^{\prime}+\Delta I_{0, F}^{\prime}+\Delta I_{0, R}^{\prime}+\Delta I_{0, C}^{\prime}\right)}}\right)
\end{aligned}
$$

where $I, I^{\prime}, I_{0}$ and $I_{0}^{\prime}$ are the intensities of the primary beam that exclude any contribution from fluorescence and scattering at positions indicated in figure 1, and $a$ and $b$ represent the efficiency of detection of the x-rays and the amplification of this signal in the downstream and upstream ion chambers, respectively. $\Delta I_{F}, \Delta I_{R}, \Delta I_{C}$ represent the contributions of the fluorescence, Rayleigh and Compton photons scattered in the forward direction and reaching the downstream ion chamber with the foil inserted into the beam. These contributions include secondary photons produced as the beam propagates through the foil and the air gap (between the foil and the downstream ion chamber). Secondary photons produced in the air gap upstream of the foil are largely absorbed by the foil and assumed not to be significant.

$\Delta I_{0, F}, \Delta I_{0, R}, \Delta I_{0, C}$ represent the contributions of the fluorescence, Rayleigh and Compton scattered photons reaching the upstream ion chambers when the foil is inserted into the beam. These contributions include secondary photons produced as the beam propagates through the air gap (between the upstream ion chamber and the foil) and through the foil itself. Secondary photons produced downstream of the target are assumed to be totally absorbed by the target and therefore are not significant.

Similar definitions apply to $\Delta I_{F / R / C}^{\prime}$ and $\Delta I_{0, F / R / C}^{\prime}$ where the prime denotes measurement without a specimen. However, in these cases, since there is no foil, the air gap is taken over the entire distance between the two ion chambers.

\subsection{Relative contributions of fluorescence, Compton and Rayleigh scattering}

Figure 3 shows the calculated percentage contributions from the photoelectric absorption, the Rayleigh and the Compton scattering cross-sections to the total mass attenuation cross-section of silver in accordance with cross-sections taken from [22]. The photoelectric absorption cross-section contributes more than $90 \%$ over the entire energy range. The contribution from Rayleigh scattering is less than $2 \%$ above the absorption edge, but reaches up to $10 \%$ below the edge. The contribution from Compton scattering is less than $1 \%$ in most of the energy range. The secondary photons arising from Compton scattering are concentrated in the high-angle region, so the effect from Compton scattered photons on the measurement of mass attenuation coefficients for silver in this energy range is negligible compared to the effect due to Rayleigh scattering and fluorescence. We therefore set all contributions from Compton scattering $\Delta I_{x, C}$ in equation (7) to be zero.

Figure 3 represents an order of magnitude estimate of the relative contributions of the various processes in the geometry of the experiment. Fluorescence photons are mainly due to $\mathrm{K}$-shell absorption, and the appropriate correction using the fluorescence yield must be applied to represent the probability of the emission of the fluorescence photons. In order to calculate the fraction of fluorescence and scattered photons reaching the ion chambers, we must also apply suitable geometrical factors representing the apertures, allow for self-absorption and for the angular distribution of the photons. 


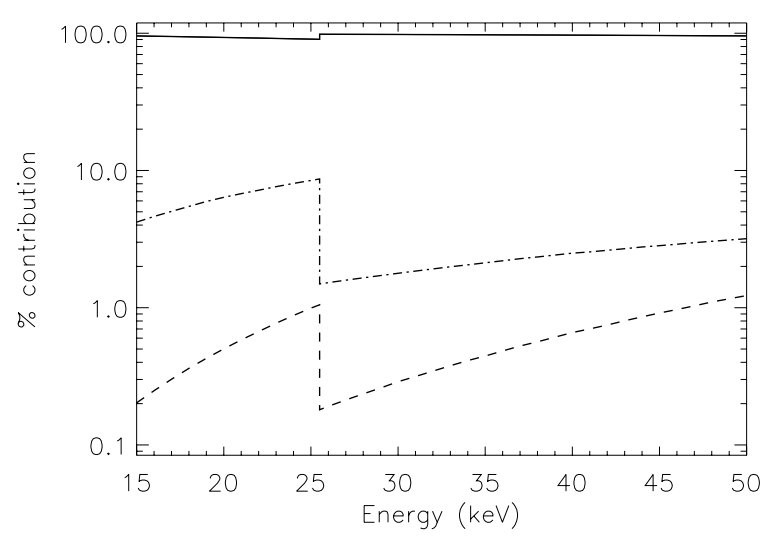

Figure 3. Calculated per cent contributions of the photoelectric absorption (solid line), Rayleigh (dot-dashed line) and Compton (dashed line) cross-sections to the total attenuation cross-section of silver. The data for the calculation are taken from [22].

\subsection{Calculation of fluorescence and Rayleigh scattering}

Consider an incident beam of intensity $I_{\text {in }}$, propagating through a medium of thickness $t_{0}$, at depths $t$ and $t+\mathrm{d} t$ from the surface. The intensities of the attenuated beam are $I_{\text {att }, t}=I_{\text {in }} \mathrm{e}^{-[\mu / \rho] \rho t}$ and $I_{\text {att }, t+\mathrm{d} t}=I_{\text {in }} \mathrm{e}^{-[\mu / \rho] \rho(t+\mathrm{d} t)}$, respectively. The total loss in intensity of the incident beam within a thin layer $\mathrm{d} t$ at depth $t$ is therefore

$\mathrm{d} I=I_{\text {att }, t}-I_{\text {att }, t+\mathrm{d} t}=I_{\text {in }} \mathrm{e}^{-[\mu / \rho] \rho t}\left[1-\mathrm{e}^{-[\mu / \rho] \rho \mathrm{d} t}\right] \approx I_{\text {in }} \mathrm{e}^{-[\mu / \rho] \rho t}[\mu / \rho] \rho \mathrm{d} t$.

The number of Rayleigh photons produced within the layer $\mathrm{d} t$ of the medium and scattered into the $4 \pi$ solid angle, is simply

$$
\mathrm{d} I_{R}=\left(\mu_{R} / \mu_{\mathrm{tot}}\right) \mathrm{d} I
$$

where $\mu_{R}$ is the (theoretically calculated) Rayleigh scattering coefficient and $\mu_{\text {tot }}$ is the total attenuation coefficient which includes photoelectric, Rayleigh and Compton scattering processes.

The deexcitation of the atom, after having absorbed an incident photon, can be either a radiative (fluorescence) or nonradiative (Auger or Coster-Kronig) process. The fluorescence yield is the probability of the emission of a fluorescence photon through the filling of a vacancy of the orbital $i$, defined as $\omega_{i}=\Gamma_{i} / \Gamma_{\text {tot }}$, where $\Gamma_{i}$ is the partial line width due to the fluorescence and $\Gamma_{\text {tot }}$ is the total line width due to fluorescence, Auger and Coster-Kronig processes. $\Gamma_{i}$ is small compared to $\Gamma_{\text {tot }}$ (except for $i$ corresponding to the K-shell), and fluorescence photons from outer shells are not penetrating, so the effect from all fluorescence photons may be approximated as being due solely to K-shell fluorescence.

The probability of the emission of a (K-shell) fluorescence photon after an incident photon has been absorbed can then be calculated to first order as $p_{F}=\omega_{i} \mu_{K} / \mu_{\text {tot }}$, where $\mu_{K}$ is the isolated $\mathrm{K}$-shell absorption coefficient [23]. The number of (K-shell) fluorescence photons produced within the layer $\mathrm{d} t$ of the medium and emitted isotropically in the entire $4 \pi$ solid angle, is therefore

$$
\mathrm{d} I_{F}=\left(\omega_{K} \mu_{K} / \mu_{\text {tot }}\right) \mathrm{d} I
$$

where $\mu_{K} / \mu_{\text {tot }}$ is the fraction of the total number of attenuated photons being absorbed by $\mathrm{K}$-shell electrons. In this analysis $\omega_{K}$ was taken from [24] and $\mu_{K}$ and $\mu_{\text {tot }}$ were taken from [25]. 


\subsection{The fraction of fluorescence and Rayleigh scattering recorded by the counters}

The effect of fluorescence and Rayleigh scattering on the ion chamber readings is then calculated from

$$
\Delta I_{x, R / F}=A \int_{0}^{t_{0}} B\left(\theta_{0}, t\right)\left(\frac{\mathrm{d} I}{\mathrm{~d} t}\right) \mathrm{d} t
$$

where $A$ is the transmission factor which takes into account absorption in the section of the beam path between the medium and the corresponding ion chamber; $B\left(\theta_{0}, t\right)$ is a correction factor which is a function of the solid angle $\theta_{0}$, the depth $t$ of the layer $\mathrm{d} t$, and the self-absorption correction within the medium.

For the secondary photons produced when the beam is propagating through the air path, $A$ is the transmission factor of the x-ray beam through a $100 \mu \mathrm{m}$ thick Kapton window of the ion chamber, which is almost equal to 1 . For the secondary photons produced inside the foil $A=A_{\text {air }} \times A_{\text {Kapton }}$, which are the transmission factors of the air paths between the target and the ion chamber and of the Kapton window, respectively:

$$
A_{\text {air }}=\exp \left[-(\mu / \rho)_{\text {air,eff }} \rho_{\text {air,eff }} t_{\text {air }}\right]
$$

where $(\mu / \rho)_{\text {air,eff }}, \rho_{\text {air,eff }}$ and $t_{\text {air }}$ are the effective mass attenuation coefficient, density and length of the air path:

$[\mu / \rho]_{\text {air }}=\frac{\% V_{\mathrm{N}_{2}} w_{\mathrm{N}_{2}}}{\% V_{\mathrm{N}_{2}} w_{\mathrm{N}_{2}}+\% V_{\mathrm{O}_{2}} w_{\mathrm{O}_{2}}}[\mu / \rho]_{\mathrm{N}}+\frac{\% V_{\mathrm{O}_{2}} w_{\mathrm{O}_{2}}}{\% V_{\mathrm{N}_{2}} w_{\mathrm{N}_{2}}+\% V_{\mathrm{O}_{2}} w_{\mathrm{O}_{2}}}[\mu / \rho]_{\mathrm{O}}$

where $w_{\mathrm{X}}$ is the molecular weight of substance $\mathrm{X}$; we have assumed air to be a mixture of $\% V_{\mathrm{N}_{2}}=78 \%$ of $\mathrm{N}_{2}$ and $\% \mathrm{~V}_{\mathrm{O}_{2}}=22 \%$ of $\mathrm{O}_{2}$ in volume.

As K-shell fluorescence is isotropic, the correction factor B in this case is calculated as

$$
B\left(\theta_{0}, t\right)=\frac{\int_{0}^{\theta_{0}} 2 \pi \sin \theta R(\theta, t) \mathrm{d} \theta}{4 \pi}=\frac{1}{2} \int_{0}^{\theta_{0}} \sin \theta R(\theta, t) \mathrm{d} \theta
$$

where $2 \pi \sin \theta \mathrm{d} \theta$ is the solid angle defined by two cones of the half-opening angles $\theta$ and $\theta+\mathrm{d} \theta$ and $R(\theta, t)$ is the self-absorption correction factor:

$$
R(\theta, t)=\exp \left[-(\mu / \rho)_{E} \rho t^{\prime} / \cos \theta\right]
$$

where $(\mu / \rho)_{E}$ is the mass attenuation coefficient of the medium at the photon energy $E, \rho$ is the density of the medium and $t^{\prime} / \cos \theta$ is the propagation distance of the X-ray photons before emerging from the boundary surface of the medium; $E$ is the energy of the secondary photon, in this case assumed to be the characteristic energy of the dominating line $E_{\mathrm{Ag}, \mathrm{K} \alpha}=22.103 \mathrm{keV}$ [15] when the incident photon energy is greater than the K-edge energy; for back-scattered radiation $t^{\prime}=t$ and for forward-scattered radiation $t^{\prime}=t_{0}-t$. While the geometrical factor $\cos \theta$ can vary significantly in the air path, for processes occurring within the medium $\cos \theta$ can be approximated as 1 , the half-opening angle $\theta_{0}(\leqslant 27 \mathrm{mrad})$ being quite small.

For the calculation of the Rayleigh scattering contribution from the foil, the angular distribution of the scattered photons must be taken into account as the process is strongly peaked in the forward direction,

$$
B\left(\theta_{0}, t\right)=\frac{\int_{\theta_{1}}^{\theta_{2}}\left(1-\cos ^{2} \theta\right) f(x, Z) \mathrm{d} \theta}{\int_{0}^{\pi}\left(1-\cos ^{2} \theta\right) f(x, Z) \mathrm{d} \theta} \mathrm{e}^{-[\mu / \rho] \rho t^{\prime}}
$$

where $\mathrm{e}^{-[\mu / \rho] \rho t^{\prime}}$ is the self-absorption correction factor in which $[\mu / \rho]$ is the mass attenuation coefficient of the absorbing medium at the energy of the incident photons; $\frac{\int_{\theta_{1}}^{\theta_{2}}\left(1-\cos ^{2} \theta\right) f(x, Z) \mathrm{d} \theta}{\int_{0}^{\pi}\left(1-\cos ^{2} \theta\right) f(x, Z) \mathrm{d} \theta}$ 


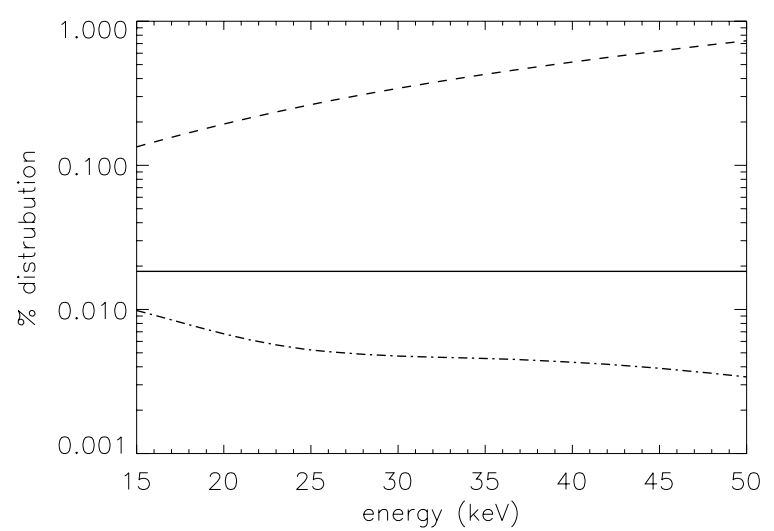

Figure 4. The percentage of photons Rayleigh-scattered within the foil, which are in the cones defined by the largest aperture in the forward (dashed line) and backward directions (dot-dashed line). The solid line represents the percentage ratio of the solid angle defined by the apertures compared to the $4 \pi$ solid angle.

represents the proportion of the Rayleigh scattered photons within the cones defined by the apertures where $\theta_{1}=0$ and $\theta_{2}=\theta_{0}$ in the forward direction and $\theta_{1}=\pi-\theta_{0}$ and $\theta_{2}=\pi$ in the backward direction.

\subsection{Results of the model calculation}

Figure 4 shows the percentage proportion of photons Rayleigh-scattered within the foil, which are in the cones defined by the largest aperture in the forward (dashed line) and backward directions (dot-dashed line). Further discussion of the calculation of the Rayleigh scattering cross-section and its angular distribution can be found elsewhere [16, 26]. Values of the atomic scattering factor $f(x, Z)$ were taken from [16]. Tabulated data were interpolated linearly on a $\log (f)$ versus $\log (x)$ scale.

It can be seen from figure 4 that the forward-scattered Rayleigh component is 1 to 2 orders of magnitude greater than the back-scattered component. Because of the geometry of the experiment, at the higher energies the effect of Rayleigh scattering becomes comparable with that from fluorescence.

Figure 5 shows the theoretical calculations of the net contribution of fluorescence and Rayleigh scattering as a percentage of the tabulated total mass attenuation coefficient $[\mu / \rho]_{\text {tab }}$ for each of the foils at the energies at which they were measured. The percentage contributions were calculated by $100 \times\left[(\mu / \rho)_{\bmod }-(\mu / \rho)_{\operatorname{tab}}\right] /(\mu / \rho)_{\operatorname{tab}}$ where $[\mu / \rho]_{\operatorname{tab}}=$ $[\mu / \rho]_{\mathrm{pe}}+[\mu / \rho]_{R}+[\mu / \rho]_{C}$. The theoretical calculation is for our experimental geometry using the largest aperture $\left(\theta_{0}=27 \mathrm{mrad}\right)$. The effect of these secondary photons is to cause the mass attenuation coefficient $[\mu / \rho]_{\bmod }$ to appear to be smaller than it would be if these photons were excluded.

As is clearly seen from the figure, the effect of secondary photons is greatest at the absorption edge where the fluorescence is strongest. It is also clearly dependent on the foil thickness used. Thicker foils show a greater effect, particularly in the region immediately above the absorption edge where the effect of the penetrating fluorescent radiation dominates. The thickness dependence of the effect becomes weaker as we move away from the edge, while the forward peaked Rayleigh scattering increases in significance. Below the edge, the 


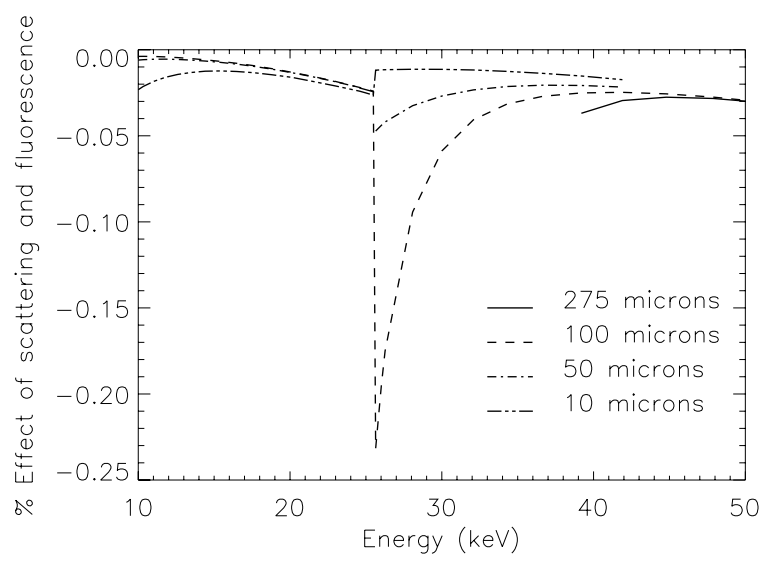

Figure 5. The effect of scattering and fluorescence on the measurement of mass attenuation coefficients $100 \times\left[(\mu / \rho)_{\bmod }-(\mu / \rho)_{\text {tot }}\right] /(\mu / \rho)_{\text {tot }}$, predicted for the largest aperture $(16 \mathrm{~mm}$ diameter). The effect of the secondary scattered photons reaching the ion chambers is to cause $[\mu / \rho]_{\text {mod }}$ to appear to be smaller than in the absence of these photons. The effect for various thicknesses is plotted at energies where those foils were used in the experiment.

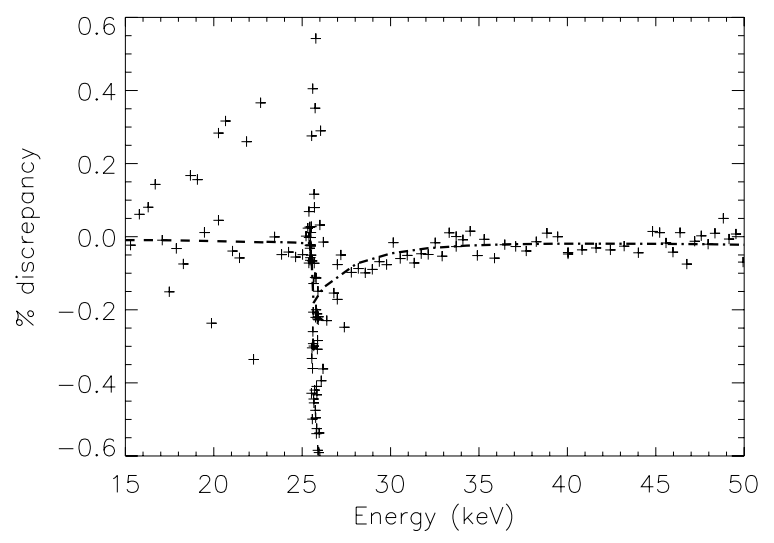

Figure 6. Per cent discrepancy between the measured mass attenuation coefficients, $[\mu / \rho]_{\text {meas }}$, measured with the largest and the medium apertures for foils located in position 3 (see figure 2). The observed discrepancies are compared with the model prediction obtained from figure 5. The dashed and dash-dot lines show the prediction for the $10 \mu \mathrm{m}$ and $100 \mu \mathrm{m}$ foils used in the data collection.

effect is due primarily to scattering and its magnitude is similar for the two thick foils (50 $\mu \mathrm{m}$ and $100 \mu \mathrm{m}$ ) used in this region.

\section{Comparison of experimental results and model calculations}

Figure 6 shows the per cent discrepancy in the measured mass attenuation coefficients, $[\mu / \rho]_{\text {meas }}$, comparing those obtained with the largest and medium apertures for foils located in position 3 (see figure 2). The observed discrepancies are compared with the model prediction (dashed line) obtained from figure 5. To obtain the magnitude of the effect on the measured $[\mu / \rho]_{\text {meas }}$ for an aperture used during the experiment, the results of figure 5 are scaled according to the corresponding solid angles. For the largest aperture (16 $\mathrm{mm}$ diameter) the scale factor 
is $(15 \times 12) /\left(\pi 8^{2}\right) \approx 0.90$, where $\left(\pi 8^{2}\right) \mathrm{mm}^{2}$ is the area of the aperture used in the model of figure 5 and $(15 \times 12) \mathrm{mm}^{2}$ is the area defined by the largest aperture and the ion chamber window of $12 \mathrm{~mm}$ height as used in the experiment; for the medium and smallest apertures the scale factors are $\left(\pi 3^{2}\right) /\left(\pi 8^{2}\right) \approx 0.14$ and $\left(\pi 1.5^{2}\right) /\left(\pi 8^{2}\right) \approx 0.04$, respectively.

The relative uncertainty in the theoretical model is estimated as about 10-20\% corresponding to absolute uncertainty in $[\mu / \rho]$ of $0.001 \%$ (away from the edge) and up to $0.3 \%$ (at the edge). This includes uncertainties in the input parameters and also in the calculation of the model. The theoretical predictions of Rayleigh, Compton scattering cross-sections, of the $\mathrm{K}$-shell fluorescence yield $\omega_{K}$ and of their angular distributions are all uncertain at the $10 \%$ level. Uncertainty in the calculation of the model is about $1 \%$ to $5 \%$, which is mainly due to geometrical simplifications.

Uncertainty in the experimental results, which is dominated by counting statistics away from the edge, is between $0.01 \%$ and $0.03 \%$ in the $23-25.2 \mathrm{keV}$ and the $27-50 \mathrm{keV}$ energy ranges. The strong XAFS modulations in $[\mu / \rho]$ as a function of energy lead to an increase in the experimental uncertainty of up to $\pm 0.5 \%$ at the edge. Below $23 \mathrm{keV}$ the experimental uncertainty is between $0.05 \%$ and $0.4 \%$. Note that throughout the $23-50 \mathrm{keV}$ range we used a single $100 \mu \mathrm{m}$ thick foil (see figure 2). This thickness provides excellent counting statistics [27], resulting in very stable measurements $( \pm(0.01-0.03 \%))$ without which observation of the absolute value of the effect of fluorescence and scattering would not have been possible. Below $23 \mathrm{keV}$, a much thinner $10 \mu \mathrm{m}$ foil was used yielding a significantly greater scatter of the data and an inability to observe the much smaller predicted $0.01 \%$ effect. Even though the $10 \mu \mathrm{m}$ foil is not ideal for studying the effect of secondary photons in this energy range, it is crucial for the investigation of other possible sources of systematics errors in the attenuation measurement (such as harmonics [31]).

At energies between $23 \mathrm{keV}$ and $50 \mathrm{keV}$ figure 6 shows remarkable agreement between the experimental results and the theoretical model. For several $\mathrm{keV}$ above the absorption edge (i.e. between $25 \mathrm{keV}$ and $30 \mathrm{keV}$ ) a significant effect due mainly to fluorescence (of up to $0.2 \%$, as predicted by the model) is clearly observed in the trend of the experimental results. Within a few hundred $\mathrm{eV}$ above the edge, the experimental data fluctuate strongly (up to $0.6 \%$ ) reflecting the strong sensitivity of the measurement to the structures of $[\mu / \rho]_{\mathrm{pe}}$ in the XAFS region. Between $30 \mathrm{keV}$ and $50 \mathrm{keV}$, effects of $0.08 \%$ to $0.03 \%$ predicted by the model are in remarkable agreement with experimental results.

Figure 7 shows a comparison, similar to that shown in figure 6, for all measurements using foils located at position 1 (see figure 2) with the largest and smallest apertures. The experimental data in figure 7 do not follow as smooth a trend as in the case of figure 6 . This is probably partly due to a small misplacement of the sample between these measurements. The poorer statistics in measurements using thinner foils at these positions also lead to larger random fluctuations in the observed discrepancy. Although thinner foils give a weaker signature for scattering and modelling, they are crucial for the investigation of other sources of experimental errors in the attenuation measurement [28, 29].

\section{Discussion}

We have demonstrated that under favourable conditions a comparison of measurements of the mass attenuation coefficient of silver taken with different-sized apertures can provide information about the effect that fluorescence and scattering have on such measurements. In particular, we show that when using thick foils in the vicinity of an absorption edge the effects are not only observable but also are in excellent accord with theoretical calculations, agreeing with them both in trend and absolute value. We emphasize that our attenuation measurements 


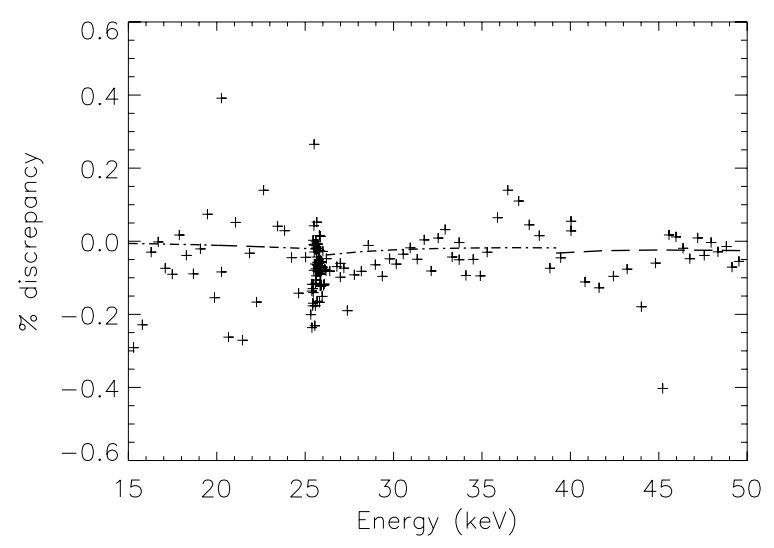

Figure 7. Per cent discrepancy between measurements of foils at position 1, comparing measurements taken with the largest and the smallest apertures. The dashed and dash-dot lines show the prediction for the 50 and $275 \mu \mathrm{m}$ foils, respectively.

have been carried out under conditions which minimize systematic errors, namely those due to local thickness determination [30], harmonic contamination [31], as well as energy calibration [32].

There is a small but clear discrepancy between the model $(0.025 \%)$ and the experimental results $(0.05 \%)$ between $23 \mathrm{keV}$ and $25 \mathrm{keV}$ as shown in figure 6 . It is intriguing that this is the region of bound-bound transitions which are not well accounted for in theoretical calculations of the photoelectric cross-section. This may be the cause of the discrepancy; alternatively, the discrepancy may be due to simplifications of the model in calculating the fluorescence effect, or to a problem with the current calculation of the angular distribution of Rayleigh scattering. These questions will require further investigation.

Conventionally, the photoelectric absorption coefficient $[\mu / \rho]_{\text {pe }}$ is often obtained experimentally by subtracting the theoretically predicted scattering cross-sections $\left(\mu_{R}+\mu_{C}\right.$ or $\left.\mu_{\mathrm{TDS}}+\mu_{C}\right)$ from the measured attenuation coefficients $[\mu / \rho]_{\text {meas }}$, which is assumed to be the (total) 'mass' attenuation coefficient $[\mu / \rho]_{\text {tot }}$. It is obvious from figures 5 and 6 that here $[\mu / \rho]_{\text {meas }}$ is always less than $[\mu / \rho]_{\text {tot }}$. The effect due to fluorescence and scattering can be significant especially in the neighbourhood of absorption edges (where fluorescence is significant) or in the high-energy region (where Rayleigh scattering is strongly peaked in the forward direction). For a reasonable collimating system $(27 \mathrm{mrad}$ for the biggest aperture in this experiment) the effect can be as large as $0.2 \%$.

This suggests that under certain conditions (i.e. poor collimation or when at high energy Rayleigh scattering is extremely peaked in the forward direction) $[\mu / \rho]_{\text {meas }}$ can be approximated as $\left([\mu / \rho]_{\text {tot }}-[\mu / \rho]_{R}\right)$, and under other conditions it is better approximated as $[\mu / \rho]_{\text {tot }}$. For critical investigations where fractions of percentages are considered to be significant this effect must be properly addressed.

Earlier measurements of attenuation, with systematic and statistical uncertainties above the $1 \%$ level, could not begin to isolate the influence of scattering processes and their angular distribution. Further, many past investigations focusing on scattering have only been able to report relative measurements or distributions, rather than confirm absolute magnitudes of coefficients for a particular process $[3,6]$. Our method of measurement involves a comparison of intensities obtained with the same detection system using the same foil. This minimizes dominant sources of error such as nonlinear counter response and harmonic contamination, 
and results in an absolute determination involving no scale factors. However, effects due to individual processes are only separated by their dependence upon energy and aperture. Additionally, angular dependences are investigated only by integration over a narrow range of angles.

More detailed observations of the angular distribution of scattering and fluorescence might be further developed. There are clear goals to obtain absolute quantification of scattering process amplitudes as well as a separation of the fluorescence, Rayleigh and Compton scattering components.

\section{Acknowledgments}

We appreciate the technical support of the APS staff, especially J Wang and P Lee. We also acknowledge the collaboration in the main attenuation measurement of D Paterson, B B Dhal and D J Cookson. This work is supported by the Australian Synchrotron Research Program which is funded by the Commonwealth of Australia under the Major National Research Facilities Program, and by a grant from the Australian Research Council. Use of the Advanced Photon Source was supported by the US Department of Energy, Basic Energy Sciences, Office of Energy Research, under contract no W-31-109-Eng-38.

\section{References}

[1] Southworth S H, Young L, Kanter E P and LeBrun T 2000 X-ray scattering and fluorescence from atoms and molecules Photoionization and Photodetachment, Advanced Series in Physical Chemistry vol 10B ed C-Y Ng (River Edge, NJ: World Scientific) pp 1289-334

[2] Jung M et al 1998 Phys. Rev. Lett. 811596

[3] Krässig B, Jung M, Gemmell D S, Kanter E P, LeBrun T, Southworth S H and Young L 1995 Phys. Rev. Lett. 754736

[4] Kanter E P, Dunford R W, Krässig B and Southworth S H 1999 Phys. Rev. Lett. 83508

[5] LeBrun T, Southworth S H, Armen G B, MacDonald M A and Azuma Y 1999 Phys. Rev. A 604667

[6] Krässig B, Dunford R W, Gemmell D S, Hasegawa S, Kanter E P, Schmidt-Böcking H, Schmitt H, Southworth S H, Weber Th and Young L 1999 Phys. Rev. Lett. 8353

[7] Mehta D, Puri Sanjiv, Garg M L and Trehan P N 1999 Phys. Rev. A 592723

[8] Rao D V, Takeda T, Akatsuka T, Seltzer S M, Hubbell J H, Cesareo R, Brunetti A and Gigante G E 2002 Phys. Scr. 65398

[9] Tran C Q, Chantler C T and Barnea Z 2003 Phys. Rev. Lett. 90257401

[10] Tran C Q, Chantler C T, Barnea Z, Paterson D and Cookson D J 2003 Phys. Rev. A 6742716

[11] Chantler C T, Tran C Q, Paterson D, Barnea Z and Cookson D J 2000 Rad. Phys. Chem. 61347

[12] Chantler C T, Tran C Q, Paterson D, Cookson D J and Barnea Z 2001 Phys. Lett. A 286338

[13] Chantler C T, Tran C Q, Barnea Z, Paterson D, Cookson D J and Balaic D X 2001 Phys. Rev. A 6462506

[14] Hubbell J H 1999 Phys. Med. Biol. 44 R1

[15] Creagh D C and Hubbell J H 1999 International Tables for Crystallography 2nd edn vol C ed A J C Wilson and E Prince (Dordrecht: Kluwer) section 4.2.4

[16] Hubbell J H and Øverbø I 1979 J. Phys. Chem. Ref. Data 869

[17] Hubbell J H, Veigele W J, Briggs E A, Brown R T, Cromer D T and Howerton R J 1975 J. Phys. Chem. Ref. Data 4471

[18] Cromer D T and Mann J B 1967 J. Chem. Phys. 471892

[19] de Marco J J and Suorti P 1971 Phys. Rev. B 41028

[20] Sano H, Ohtaka K and Ohtsuki Y H 1969 J. Phys. Soc. Jpn. 271254

[21] Gerward L and Thuesen G 1977 Z. Naturforsch 32a 588

[22] Berger M J and Hubbell J H 1987 XCOM:Photon Cross Sections Database (NBSIR 87-3597, NIST) Berger M J and Hubbell J H 1999 XCOM: Photon Cross Sections Database version 1.2 http://physics. nist. gov/ xcom

[23] Chantler C T 1995 J. Phys. Chem. Ref. Data 2471 
[24] Bambynek W, Crasemann B, Fink R W, Freund H U, Mark H, Swift C D, Price R E and Rao P V 1972 Rev. Mod. Phys. 44716

[25] Chantler C T 2000 J. Phys. Chem. Ref. Data 29597

Chantler C T, Olsen K, Dragoset R A, Kishore A R, Kotochigova S A and Zucker D S 2003 X-Ray Form Factor, Attenuation and Scattering Tables version 2.0 http://physics. nist. gov/ffast

[26] Kissel L, Pratt R H and Roy S C 1980 Phys. Rev. A 221970

[27] Chantler C T, Tran C Q, Paterson D, Barnea Z and Cookson D J 2000 X-ray Spectrom. 29449

Chantler C T, Tran C Q, Paterson D, Barnea Z and Cookson D J 2000 X-ray Spectrom. 29459

[28] Creagh D C 1999 International Tables for Crystallography 2nd edn vol C ed A J C Wilson and E Prince (Dordrecht: Kluwer) section 4.2.3 p 213

[29] Chantler C T, Barnea Z, Tran C Q, Tiller J B and Paterson D 1999 Opt. Quantum Electron. 31495

[30] Tran C Q, Chantler C T, Barnea Z and de Jonge M D 2003 Rev. Sci. Instrum. at press de Jonge M D, Barnea Z, Tran C Q and Chantler C T 2004 Meas. Sci. Technol. 151811

[31] Tran C Q, Barnea Z, de Jonge M D, Dhal B B, Paterson D, Cookson D J and Chantler C T 2003 X-ray Spectrom. 3269

[32] Chantler C T, Tran C Q and Cookson D J 2004 Phys. Rev. A 69042101 